\title{
光电协同催化有机合成研究进展
}

\author{
杨 光 王衍伟仇友爱* \\ (南开大学化学学院 元素有机化学国家重点实验室 天津 300071)
}

\begin{abstract}
摘要 随着有机电化学和光化学的复兴, 将两者结合的新型光电协同催化有机化学成为了研究热点之一. 光电协同催 化广泛应用于氧化还原及偶联反应等众多反应体系. 本综述总结了近 5 年来光电催化的最新研究进展, 对反应的机理 及催化剂进行了系统性的分类，总结了该类反应体系的优势和特点，并且对该研究方向的发展进行了展望.
\end{abstract}

关键词 有机电化学; 光化学; 光电化学; 协同催化

\section{Advances in Organic Photoelectrochemical Synergistic Catalysis}

\author{
Yang, Guang Wang, Yanwei Qiu, Youai* \\ (State Key Laboratory of Elemento-organic Chemistry, College of Chemistry, Nankai University, Tianjin 300071)
}

\begin{abstract}
Following the renaissances of organic electrochemistry and photochemistry, photoelectrochemical approach was arising as one of hot research areas by combination advantages of photocatalysis and electrocatalysis. The photoeletrocatalysis was widely applied in redox reactions, coupling reactions and beyond. The development of photoelectrochemical transformations during past five years is summarized and the related reactions are classified according to mechanism and catalyst. The current progresses and future outlooks of such method are also discussed.
\end{abstract}

Keywords organic electrochemistry; photochemistry; photoelectrochemistry; synergistic catalysis

光作为一种来源广泛、可再生、无污染的能源, 一 直以来, 将光应用于有机合成反应受到了许多科学家的 关注. 早在 1912 年, Ciamician ${ }^{[1]}$ 就提出使用光能作为廉 价的化学合成试剂. 自 20 世纪 70 年代以来, 利用光氧 化还原催化引发有机转化引起了人们的极大兴趣. 一般 来说, 光氧化还原催化过程主要通过金属络合物如 $\mathrm{Ru}^{\mathrm{II}[2-3]} 、 \mathrm{Ir}^{\mathrm{III}}{ }^{[4-6]}$ 或有机染料 ${ }^{[7-8]}$ 等光敏剂在可见光激发下 与有机底物进行单电子转移(Single Electron Transfer, SET) 来实现. 光催化反应的出现有力地推动了绿色有 机合成的发展, 而且在成本节约、安全性提高和环境友 好性等方面有着重要意义 ${ }^{[9-11]}$. 与此同时, 电化学作为 另外一种单电子转移(SET)方法, 在 19 世纪 30 年代, Faraday 首先开始了电化学研究, 受其启发, Kolbe 在 1847 年发展了经典的 Kolbe 电解反应, 提供了一种简单 的烷基羧酸脱羧偶联生成烷烃的方法. 电化学因成本 低, 条件易于调节等特点在近年来也受到了广泛的关 注 ${ }^{[12-22]}$.
在过去的数十年中, 能源和环境危机日益突出, 亟 需绿色有机化学方法的出现. 作为有效的单电子转移 (SET)方法, 光催化和电催化在有机合成中受到了越来 越多化学家的关注. 但是长期以来, 这两种方法的联合 使用一直未受到足够的关注. 1979 年, Moutet 和 Reverdy 课题组 ${ }^{[23]}$ 首次提出了电光化学的概念, 他们对电化学 氧化产生的吩噻嗪(PTZ)自由基阳离子 $\mathrm{PTZ}^{+}$进行可见 光激发, 可以将 1,1 -二苯乙烯的苯基氧化为自由基正离 子, 同时使 PTZ 再生. 1,1-二苯乙烯被氧化得到的自由 基阳离子可以与另一分子的 1,1-二苯乙烯进行 $[4+2]$ 环 加成或 1,2-加成, 中间体与水进一步发生反应最终得到 化合物 1-2 或 1-3 (图 1A). 1982 年, Moutet 和 Reverdy 课 题组接着报道了一种光电催化氧化反应，电产生的 $N, N, N^{\prime}, N^{\prime}$-四苯基对苯二胺(TPPD)自由基阳离子在紫外 光照下被激发产生的 ${ }^{*} \mathrm{TPPD}^{+}{ }^{+}$能使茮醇氧化为苯甲醛 1-5 ${ }^{[24]}$ (图 1B). 1983 年, Sheffold 课题组 ${ }^{[25]}$ 报道了 $\alpha, \beta$-不 饱和羰基化合物与酰基的光电化学 1,4-加成反应. 维生

\footnotetext{
* Corresponding author. E-mail: qiuyouai@nankai.edu.cn

Received May 31, 2021; revised June 25, 2021; published online July 5, 2021.

Dedicated to the 100th anniversary of Chemistry at Nankai University.

Project supported by the Fundamental Research Funds for the Central Universities (No. 63213063).

中央高校基本科研业务费专项资金(No. 63213063)资助项目.
} 
素 $\mathrm{B}_{12 \mathrm{a}}\left(\mathrm{Co}^{\mathrm{III}}\right)$ 作为该反应的催化剂, 在电流和光的作用 下发生还原和裂解，导致了酰基自由基的生成，该自由 基与 $\alpha, \beta$-不饱和羰基化合物发生 1,4-加成反应得到 1-8 (图 1C).

(A)
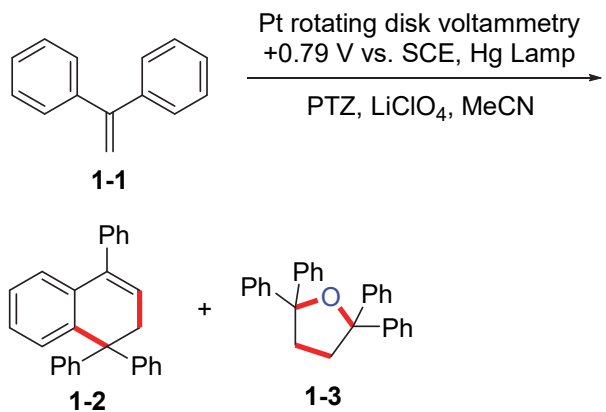

(B)

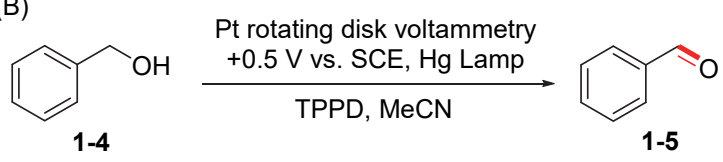

(C)

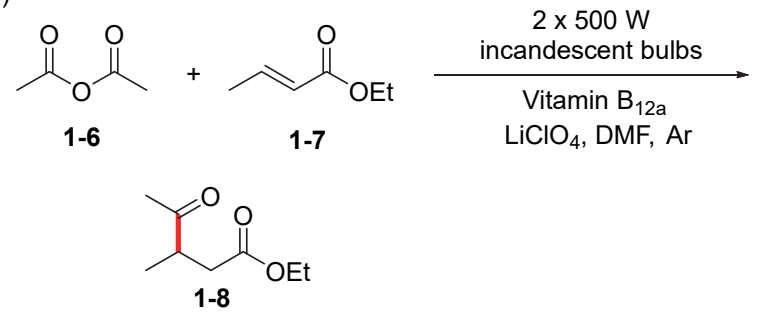

图 1 早期光电催化反应

Figure 1 Original electrophotocatalyzed transformations

最近几年, 随着现代有机电化学的发展, 独特的光 电协同催化方法引起了科学家们广泛关注. 多个课题组 在光电催化有机合成方面开展了系统的工作, 基于光电 协同催化开发出了一系列高效的催化氧化还原、偶联反 应等. 使用不同光电催化剂, 例如高氧化性或还原性光 电催化剂(图 2), 代替化学氧化剂或还原剂, 同时还可以 利用具有较高氧化性或还原性的催化剂进行惰性键活 化研究, 为有机合成化学提供了一种新型的合成策略. 本综述总结了该领域的最新研究成果, 根据其反应历程 等对目前报道的光电催化有机反应进行了如下分类: (1) 电化学生成离子的光激发过程; (2)电代替氧化还原剂; (3)解耦光电化学; (4)界面光电化学; (5)流动光电化学. 其中(1)和(2)在一些综述中被统称为电化学介导光氧化 还原催化 ${ }^{[26-28]}$.

\section{1 光电催化反应}

\section{1 电化学生成离子的光激发}

电氧化还原与光激发作用于同一个化合物以生成 高氧化还原电位的催化剂是光电化学最常见的方法之
一。这种 “光电催化剂” 可以同时利用光能和电能，能 够作为这种催化剂需要满足以下条件: (1)能够在电流的 作用下发生单电子氧化/还原产生稳定的中间体; (2)被 氧化/还原后的中间体可以吸收可见光以生成激发态; (3)激发态具有足够长寿命以与底物发生反应; (4)催化 剂可以在整个催化循环中保持稳定.

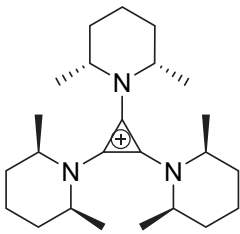

$\mathrm{TAC}^{+}$<smiles>N#Cc1c2ccccc2c(C#N)c2ccccc12</smiles>

DCA

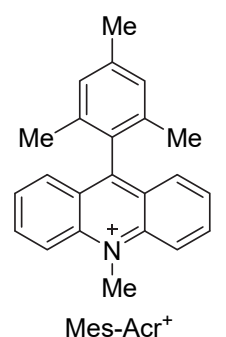

Mes-Acr ${ }^{+}$<smiles>CC(=O)OCC(OC(C)=O)C(OC(C)=O)C(Cn1c2nc(=O)[nH]c(=O)c-2nc2cc(C)c(C)cc21)OC(C)=O</smiles>

图 2 常见光电催化剂示例
Figure 2 Selected examples of electrophotocatalysts

2019 年, Lambert 课题组 ${ }^{[29]}$ 开发了一类基于三氨基 环丙烯离子 $\left(\mathrm{TAC}^{+}\right)$的光电催化剂. 无色的基态 $\mathrm{TAC}^{+}$在 阳极被氧化为双阳离子自由基 $\left(\mathrm{TAC}^{\cdot 2+}\right.$ ), 该阳离子自由 基吸收可见光后成为激发态的 ${ }^{*} \mathrm{TAC}^{\cdot 2+}\left(E_{1 / 2}=+3.33 \mathrm{~V}\right.$ vs. SCE). 该催化剂可在 $1.5 \mathrm{~V}$ 电压的条件下氧化未激活 的芳基化合物形成相应的阳离子自由基，实现与唑类化 合物的偶联反应. 机理研究表明, 被激发态 ${ }^{*} \mathrm{TAC}^{\cdot 2}$ 氧化 后的苯基阳离子自由基中间体 3-4 与吡唑 3-2 发生亲核 加成反应，产生的芳基自由基中间体 3-5 被阳极或者 $\mathrm{TAC}^{\cdot 2+}$ 氧化并失去质子芳构化后, 得到目标产物 3-3. 在阴极发生了氢离子的还原, 产生 $\mathrm{H}_{2}$, 光电催化剂 ${ }^{*} \mathrm{TAC}^{\cdot 2+}$ 在氧化苯基后重新生成基态 $\mathrm{TAC}^{+}$(图 3).

进一步研究发现, TAC 还可用于醚类的 $\alpha$ 位官能团 化 ${ }^{[30]}$, 使醚与异喹啉、烯烃、炔烃、吡唑和嘌呤进行无 氧化剂偶联. 该体系对于小位阻的醚的 $\alpha$ 位具有较高的 区域选择性. 机理研究表明, TAC 在该反应中起着氢原 子转移(HAT)催化剂的作用. $\mathrm{TAC}^{+}$经阳极氧化和光激发 后生成的激发态二价阳离子与醚底物 4-1 发生氢原子转 移(HAT), 生成相应的自由基中间体 4-4 和质子化的 $\mathrm{TAC}^{2+}$. 生成的自由基中间体 4-4 与异喹啉 4-2 反应生成 自由基中间体 4-5, 随后进行第二次氧化并失去质子以 生成产物 4-3. 同时，二价阳离子 $\mathrm{TAC}^{2+}$ 去质子化使 $\mathrm{TAC}$ 催化剂再生以完成催化循环. 相较于该方法, 直接 

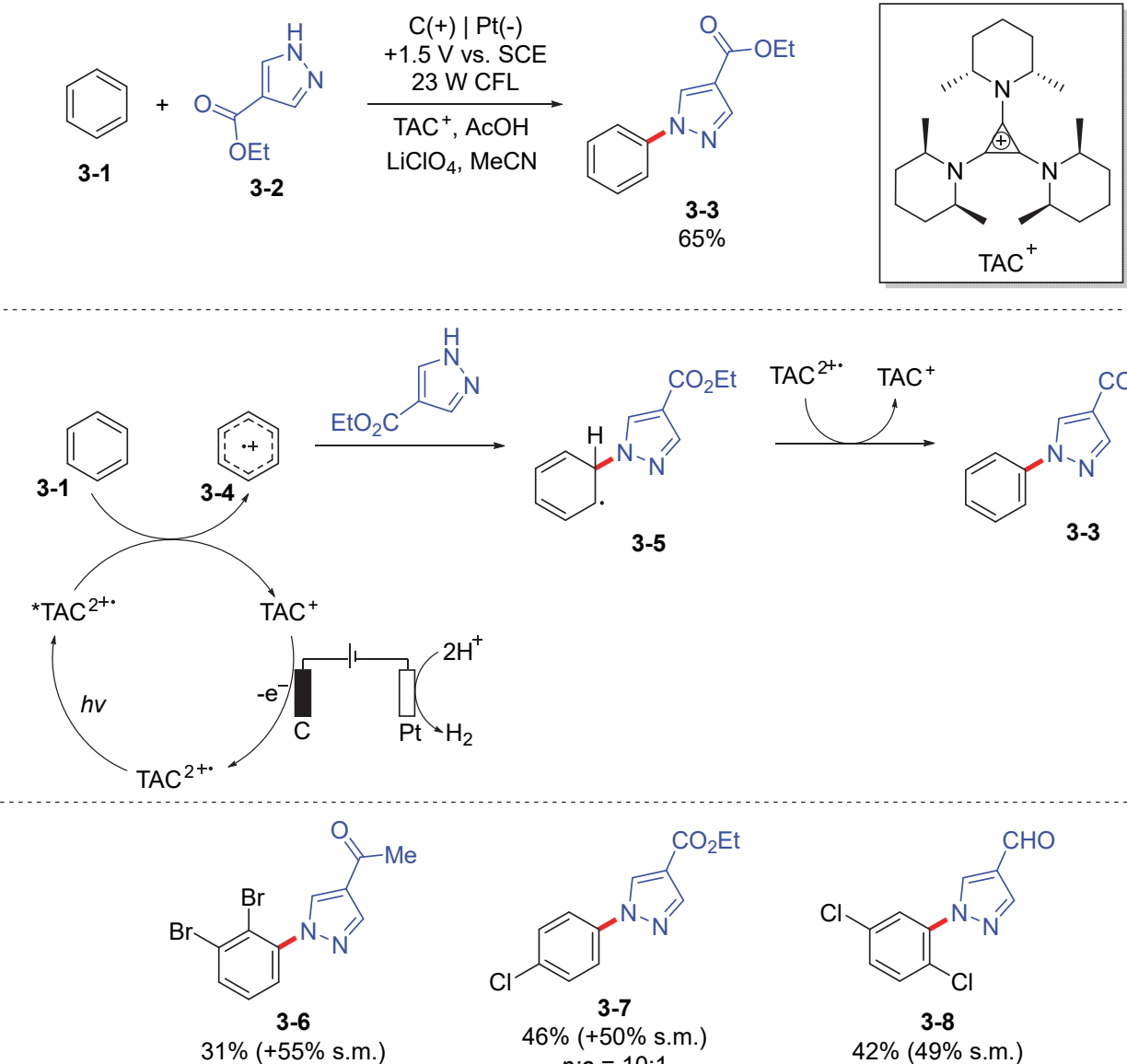

图 3 三氨基环丙烯离子催化的芳烃与氮杂芳烃氧化偶联反应

Figure 3 Trisaminocyclopropenium catalyzed oxidative coupling of arenes with nitrogen heteroaromatics

电解来实现醚类的官能团化则相对困难，因为自由基中 间体比底物更容易被氧化, 而阳极提供了持续氧化的能 力, 容易生成副产物. 相比之下光电催化策略仅保持低 浓度的活性氧化剂, 可以延长自由基的存在时间, 使其 可以进行单电子化学反应生成目标产物(图 4).

最近, Lambert 课题组 ${ }^{[31]}$ 使用该光电催化剂催化 Ritter 反应, 实现了烷基邻近 $\mathrm{C}-\mathrm{H}$ 键的双氨化反应. 该 反应首先通过 $\mathrm{TAC}$ 催化苄基位点进行 Ritter 反应, 光激 发的 TAC 自由基二价阳离子对底物进行单电子氧化产 生自由基阳离子 5-3, 然后经过去质子化和二次氧化得 到阳离子中间体 5-4, 溶剂化后生成 Ritter 反应产物 5-5. 在标准反应条件下, 乙酰胺 5-5 将转化为二氢咪唑产物 5-2, 但是第二次 $\mathrm{C}-\mathrm{H}$ 键氨基化的途径尚不确定. 作者 推测 Ritter 产物可能经历可逆的酸催化消除反应, 生成 $\alpha$-甲基苯乙烯 5-6, 后者经单电子氧化、溶剂捕获、氧化 得到二氢咪唑产物 5-2(图 5).

除了氧化反应外, 光电催化也可用于生成强还原性 的物种, 应用于还原反应. Lambert 和 Lin 课题组 ${ }^{[32]}$ 报道 了使用光电激发生成 9,10-二氧基葱基激发态自由基阴 离子 ${ }^{*} \mathrm{DCA}^{{ }^{-}}\left(E_{1 / 2}=-3.2 \mathrm{~V}\right.$ vs. SCE $)$ 进行的还原偶联反
应。该催化剂可用于活化具有较高还原电位的底物 $\left(E_{\mathrm{red}} \approx-1.9 \sim-2.9 \mathrm{~V}\right)$, 如氯代或溴代芳烃, 产生的芳 基卤化物自由基阴离子 6-3 经裂解得到卤负离子和芳基 自由基 6-4, 后者可被 $\mathrm{B}_{2} \mathrm{pin}_{2}, \mathrm{Sn}_{2} \mathrm{Me}_{6}$ 或杂芳烃捕获，生 成芳基硼酸酯 6-2、芳基锡烷 6-5 或联芳基 6-7 等产物. 阳极发生锌电极的氧化. DCA ${ }^{-}$由多孔碳电极通过 DCA 的阴极还原而产生，计算结果表明，该自由基阴离子的 高还原电位是由于 SOMO-HOMO 能级反转引起的. 相 较于强还原性金属( $\mathrm{Li}$ 和 $\mathrm{Na}$ 等)或直接电解产生的强还 原条件，电化学活化和光激发偶联可以可控地以非常低 的浓度形成还原催化剂, 表现出较好的化学选择性. 同 时，Pd 催化也可用于该硼化官能化反应获得相似产物, 但是当偶联底物包含路易斯碱基团时受配位作用影响, 产率会大幅度降低, 因此该光电协同方法具有显著优势 (图 6).

\section{2 电代替氧化还原剂}

替代氧化还原剂是光电协同催化的另一个非常重 要的应用. 传统光化学催化通常需要牺牲氧化还原剂, 在反应中容易产生副产物, 而氧化还原剂本身及其副产 物可能与反应原料或产物发生反应，使反应体系复杂 

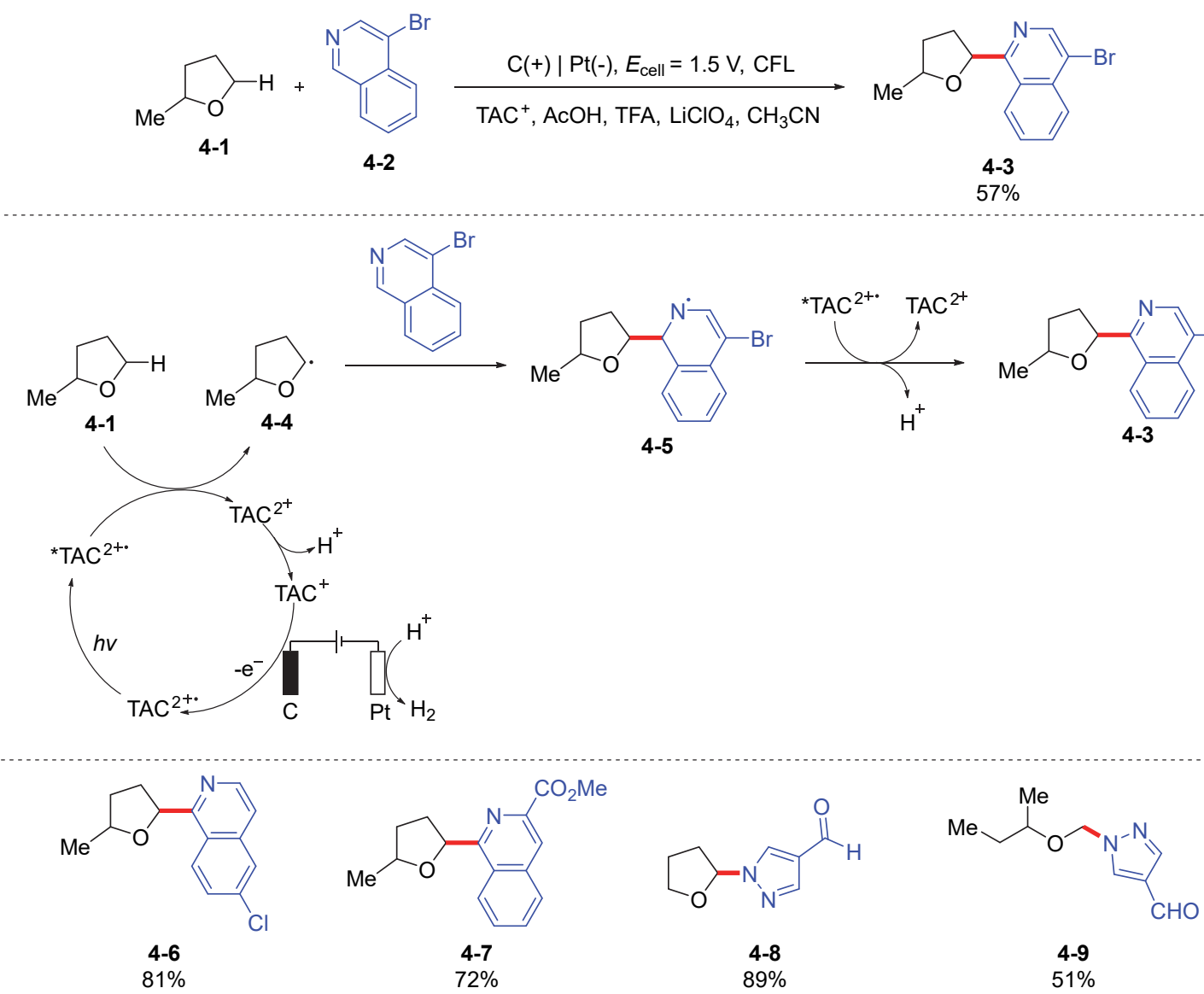

图 $4 \mathrm{TAC}$ 催化的醚 $\alpha$ 位的 $\mathrm{C}-\mathrm{H}$ 官能团化反应

Figure 4 TAC catalyzed $\mathrm{C}-\mathrm{H}$ functionalization of ethers at $\alpha$-position

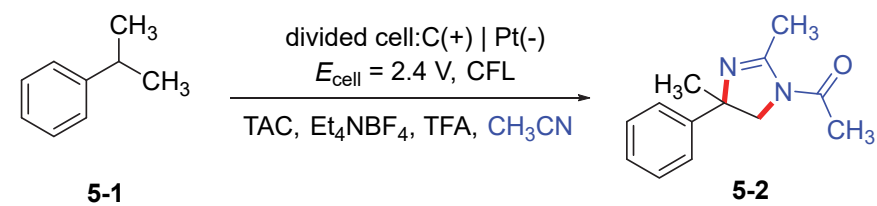

$5-1$

$\mathrm{CH}_{3}$

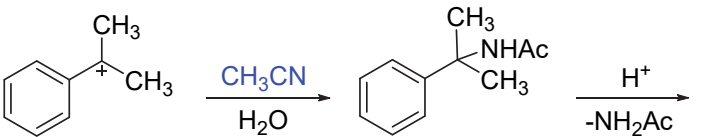

5-5<smiles>C=C(C)c1ccccc1</smiles>

5-6
5-1

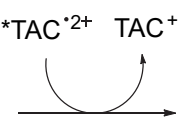

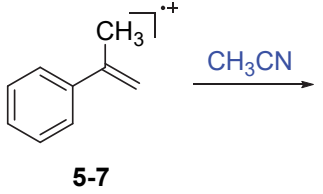

$5-7$

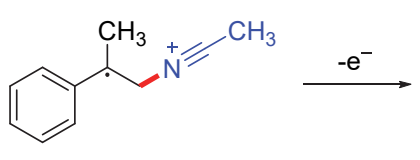

$5-8$

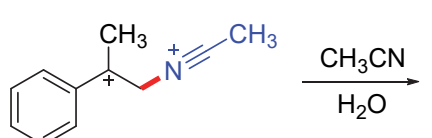

5-9

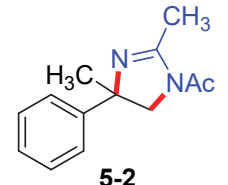

$5-2$

图 5 光电催化邻位烷基芳烃双氨化

Figure 5 Electrophotocatalytic diamination of vicinal $\mathrm{C}-\mathrm{H}$ bonds

化, 化学计量的氧化还原剂也不利于大规模反应应用. 用电代替氧化还原剂, 符合原子经济性的要求, 从工业 界的角度来说也具有一定的应用价值.

2019 年, 徐海超课题组 ${ }^{[33]}$ 实现了电调控光催化杂 环芳烃与三氟硼酸酯化合物的 $\mathrm{C}-\mathrm{H}$ 烷基化反应. 9-均 三甲基苯基-10-甲基唤啶(Mes-Acr ${ }^{+}$)是一种有机染料,
经光激发生成具有强氧化性的激发态 $\mathrm{Mes}-\mathrm{Acr}^{+*}\left(E_{\mathrm{red}}=\right.$ $2.06 \mathrm{~V}$ vs. SCE), 其与烷基三氟嗍酸之间发生单电子转 移，生成唤啶基自由基 Mes-Acr 和烷基自由基 $\mathrm{R}^{\circ}$. 烷基 自由基和质子化后的喹啉发生 Minisci 反应, 生成目标 产物 7-6. 而自由基 Mes-Acr・则在阳极被氧化为基态 Mes-Acr ${ }^{+}$. 该反应底物范围广泛，包括异喹啉、菲啶、 


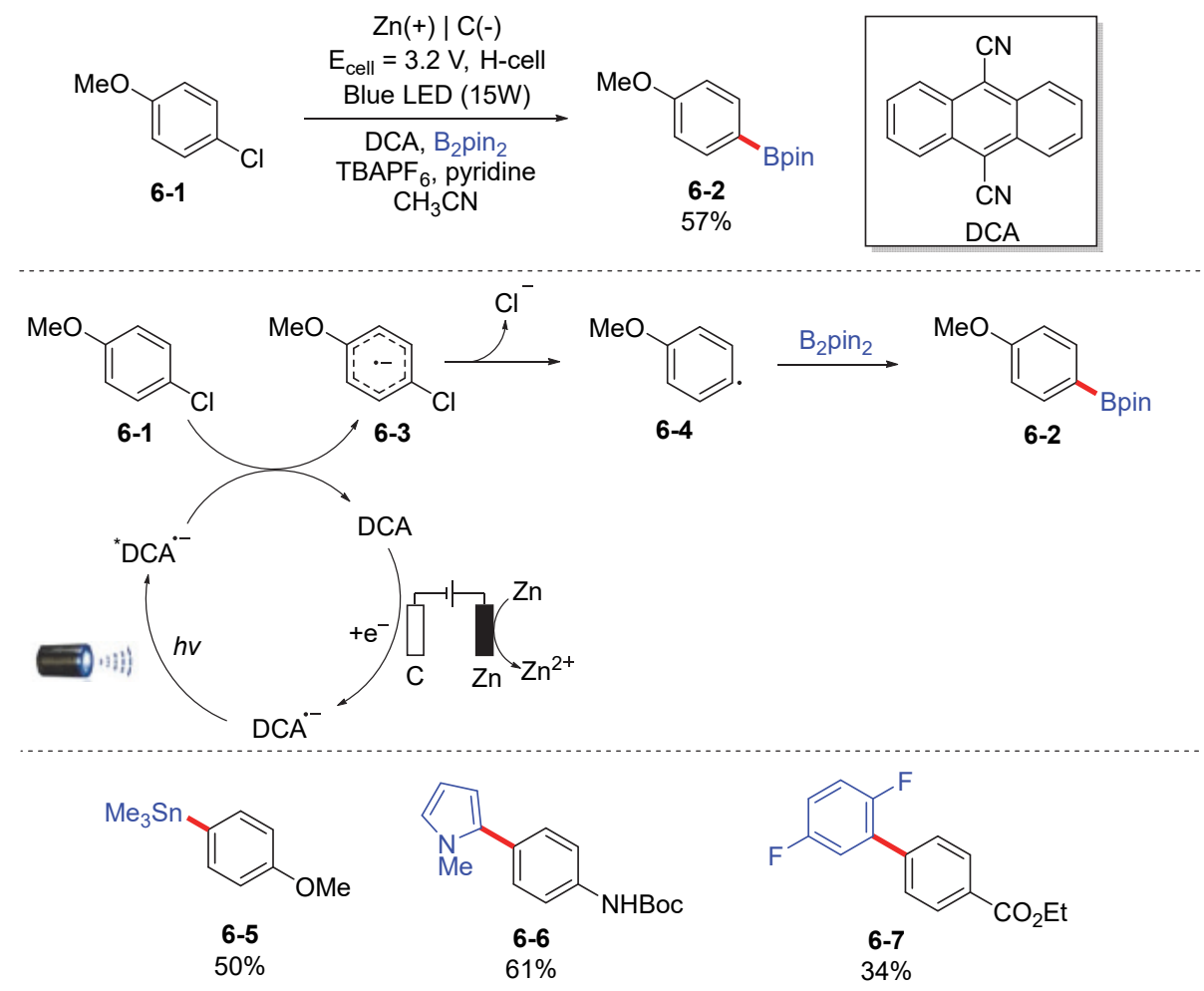

图 6 双氰基葱自由基阳离子催化的芳基卤化物还原偶联反应

Figure 6 Dicyanoanthracene radical cation catalyzed reduction coupling of aryl halides

酞嗪、苯并噻唑、鲐啶和嘌呤等都可以适用(图 7).

2020 年, 徐海超课题组 ${ }^{[34]}$ 使用光电催化实现了杂 芳烃和 $\mathrm{C}\left(\mathrm{sp}^{3}\right)-\mathrm{H}$ 在无金属催化剂或化学氧化剂的条件 下的高效脱氢交叉偶联. 首先. $\mathrm{Cl}^{-}$通过阳极氧化生成 $\mathrm{Cl}_{2}$, 随后在光照条件下 $\mathrm{Cl}_{2}$ 发生均裂, 生成 $\mathrm{Cl}^{-}$自由基. 然后, 与之发生氢原子转移(HAT)产生的亲核碳自由基 加成到质子化的 $\mathrm{N}$-杂芳香族化合物中, 生成自由基阳 离子中间体 8-4, 然后借助 $\mathrm{Cl}^{\circ}$ 进行重新芳构化(图 8).

同一年, Ackermann 课题组 ${ }^{[35]}$ 使用 Mes-Acr ${ }^{+}$实现了 光电催化 $\mathrm{C}\left(\mathrm{sp}^{2}\right)-\mathrm{H}$ 三氟甲基化反应. ${ }^{*} \mathrm{Mes}-\mathrm{Acr}^{+}$氧化 $\mathrm{CF}_{3} \mathrm{SO}_{2}{ }^{-}$生成的 $\mathrm{CF}_{3} \mathrm{SO}_{2}$ 自由基失去一份子 $\mathrm{SO}_{2}$ 后产生 $\mathrm{CF}_{3}$ ・自由基，随后对芳基底物进攻形成自由基中间体 9-4, 而 Mes-Acr 则被阳极氧化为 Mes-Acr ${ }^{+}$, 自由基中 间体 9-4 经过 Mes-Acr ${ }^{+}$单电子转移氧化形成阳离子络合 物 9-5. 同时, 9-4 也可以在阳极处被直接氧化. 最后, 9-5 去质子后得到目标产物. 阴极发生质子还原反应生成 $\mathrm{H}_{2}$. 该反应可适用于广泛的底物, 除了吸电子或给电子 基的芳烃外, 其他杂环如呋喃、噻吩、苯并呋喃、苯并 噻吩、吲哚、喹啉和嘧啶等杂芳烃都可以在该反应体系 下发生三氟甲基化反应(图 9).

最近, 徐海超课题组 ${ }^{[36]}$ 报道了使用 $\mathrm{Mes}^{-} \mathrm{Acr}^{+}$与 TEMPO 组成的双催化体系催化芳烃与唑类化合物脱氢 交叉偶联反应. Mes-Acr ${ }^{+}$光激发产生的高度氧化的激发
态 ${ }^{*}$ Mes-Acr ${ }^{+}$将二苯醚氧化为芳烃自由基阳离子中间体 10-4, 同时形成自由基 Mes-Acr。中间体 10-4 被吡唑捕 获，在失去质子后生成环己二烯基自由基 10-5, 后者通 过氢原子转移(HAT)与 TEMPO 反应, 生成最终的 $\mathrm{C}-\mathrm{H}$ 官能化产物 10-3 和 TEMPO-H. 自由基 Mes-Acr 和 TEMPO-H 在阳极被氧化, 再生催化剂 Mes-Acr ${ }^{+}$和 TEMPO. 在阴极处, 质子被还原生成 $\mathrm{H}_{2}$. 反应产物可 与多种取代的苯相容，如联苯、均三甲苯、各种烷氧基 苯等，且单取代苯在反应中都表现出区域选择性，萗、 甲基吡唑等也可与吡唑发生 $\mathrm{C}-\mathrm{H}$ 官能团化反应. 在该 反应体系中，咪唑无法与均三甲苯发生偶联，可能由于 反应依赖于芳烃自由基阳离子的形成来形成 $\mathrm{C}-\mathrm{N}$ 键, 所以芳烃需要在比吡唑更低的电势被氧化才能发生交 叉偶联反应，因此该电光催化方法的芳烃范围受到鲐啶 催化剂的氧化还原电势 $\left(E^{\mathrm{red}}=2.06 \mathrm{~V}\right.$ ) 的限制(图 10).

2020 年, Lambert 课题组 ${ }^{[37]}$ 报道了光电催化未活化 的芳基氟化物在常温下的亲核芳香取代反应 $\left(\mathrm{S}_{\mathrm{N}} \mathrm{Ar}\right)$, 2,3-二氯-5,6-二氰基醌(DDQ)作为催化剂, 吸收光子后 产生的激发态 ${ }^{*} \mathrm{DDQ}\left(E_{\mathrm{red}}=3.18 \mathrm{~V}\right.$ vs. SCE) 可以氧化芳基 氟化物生成阳离子自由基中间体 11-4，接着 11-4 被吡唑 亲核进攻得到自由基中间体 11-5, 该中间体在阴极以单 电子转移(SET)方式还原, 氟离去得到目标产物 3. 被底 物还原的 $\mathrm{DDQ}^{--}$在阳极被氧化恢复基态 DDQ. 值得注 
<smiles>Cc1ccnc2ccccc12</smiles>

7-1<smiles>[13CH3]C([13CH3])[13CH3]</smiles>

7-2
$\mathrm{C}(+) \mid \mathrm{Pt}(-),+1.5 \mathrm{~V}$ vs. SCE $20 \mathrm{~W}$ blue LED
Mes-Acr ${ }^{+}$, TFA, MeCN/ $\mathrm{H}_{2} \mathrm{O}$

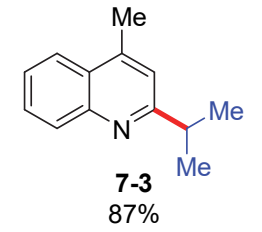

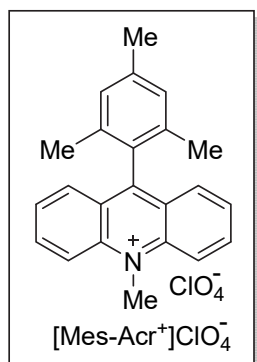
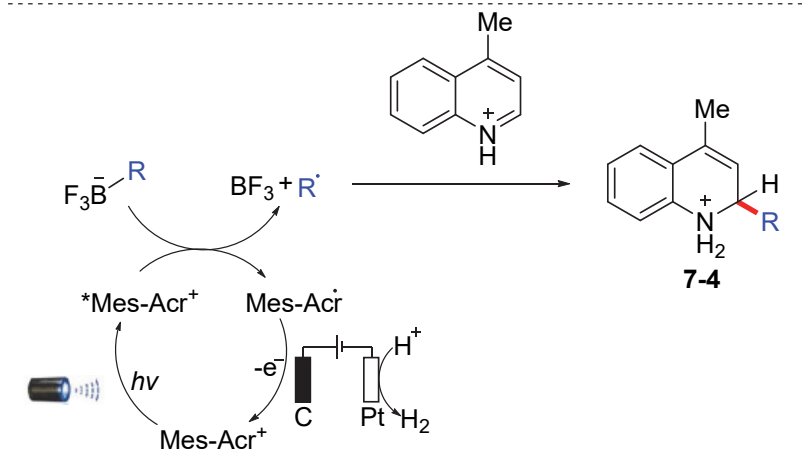<smiles>[R]C1C=C(C)c2ccccc2N1</smiles>

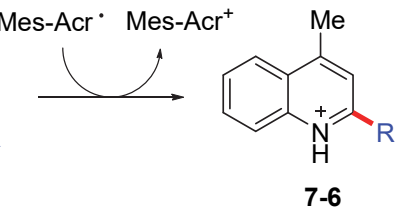

7-6<smiles>CC(C)c1c2ccccc2nc2ccccc12</smiles>

7-7<smiles>CC(C)c1nc2cc(Br)ccc2s1</smiles>

$7-8$

$78 \%$

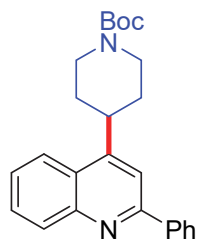

7-9

$70 \%$

图 7 光电催化杂芳烃与三氟嗍酸酯的 $\mathrm{C}-\mathrm{H}$ 烷基化反应

Figure 7 Electrophotocatalyzed $\mathrm{C}-\mathrm{H}$ alkylation of heteroarenes with organotrifluoroborates<smiles>c1ccc(-c2ccc3ccccc3n2)cc1</smiles>

8-1
8-2

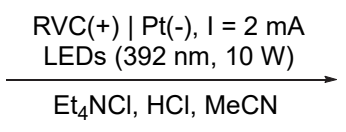

$\mathrm{Et}_{4} \mathrm{NCl}, \mathrm{HCl}, \mathrm{MeCN}$

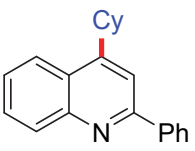

8-3

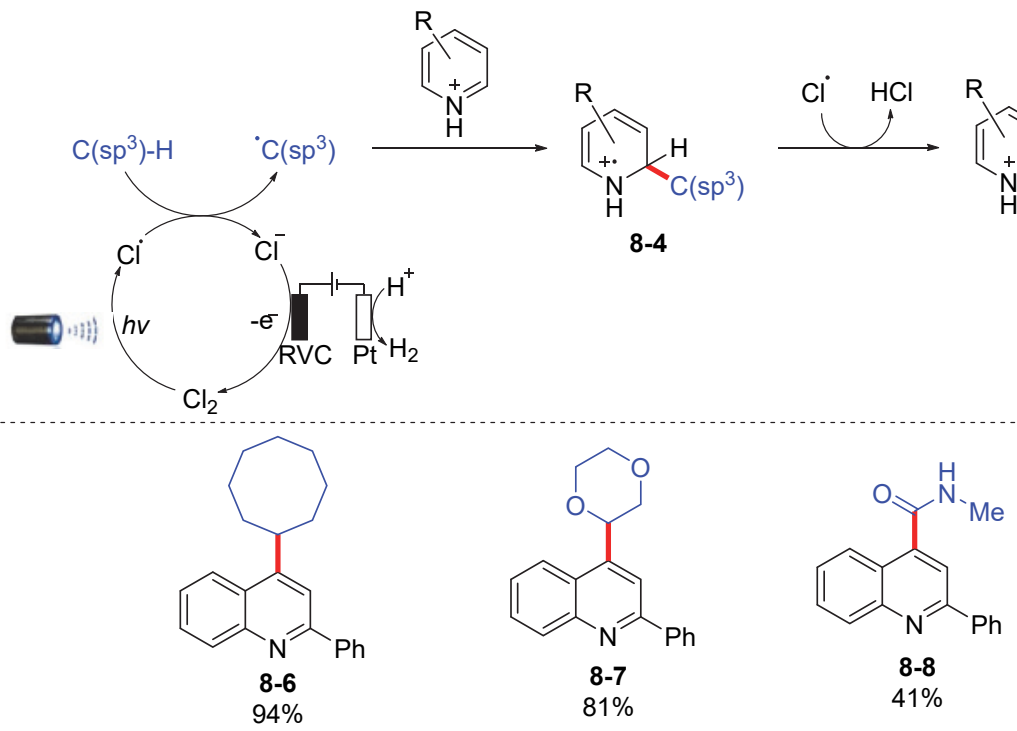

图 8 光电催化杂芳烃与脂肪烃 $\mathrm{C}-\mathrm{H}$ 交叉偶联反应

Figure 8 Electrophotocatalyzed cross-coupling of heteroarenes with aliphatic $\mathrm{C}-\mathrm{H}$ bonds 

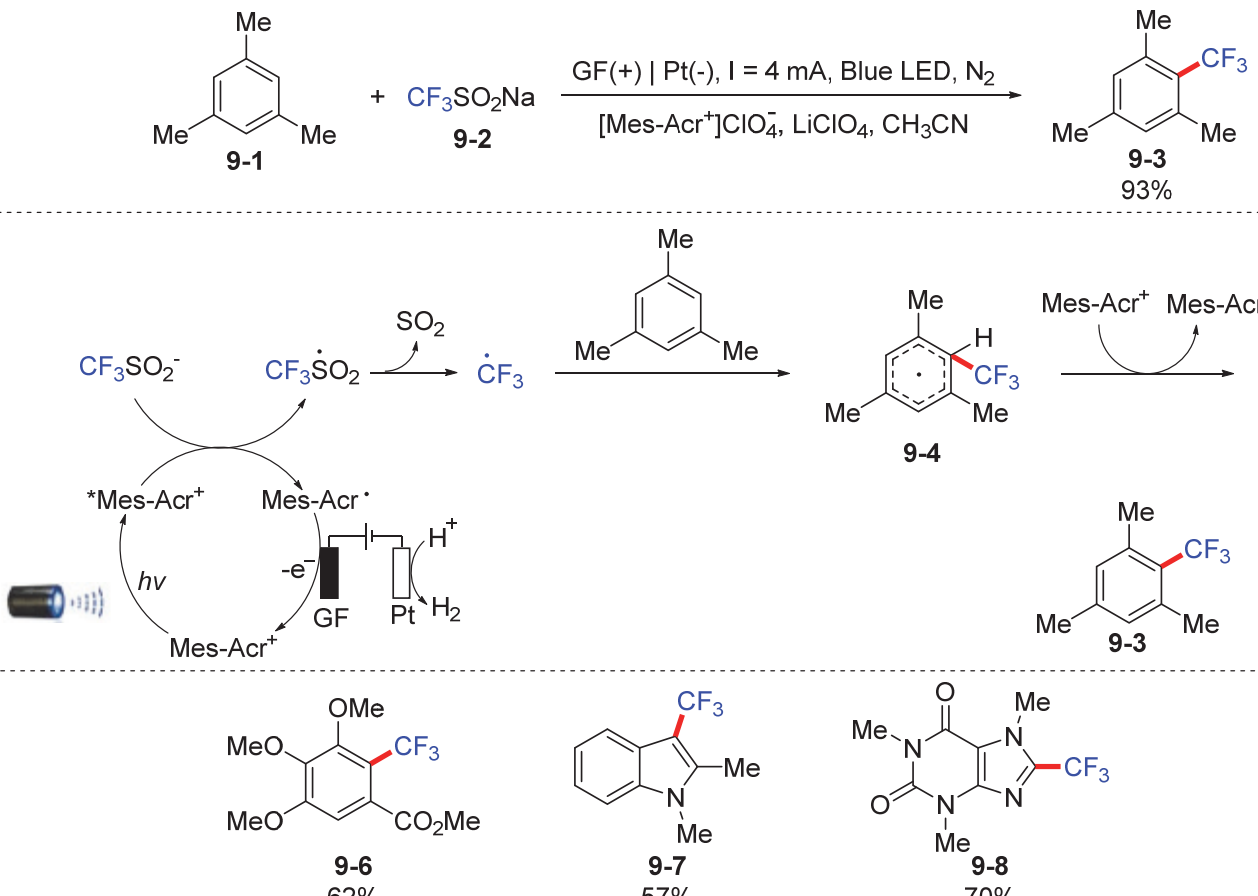<smiles>Cc1cc(C)cc(C)c1</smiles><smiles>Cc1cc(C)c(C(F)(F)F)c(C)c1</smiles><smiles>CC(C)(Cl)C[Ge](C)(C)C</smiles><smiles>Cc1cc(C)c(C(F)(F)F)c(C)c1</smiles><smiles>Cc1cc(C)c(C(F)(F)F)c(C)c1</smiles>
9-5 $\mathrm{H}^{+}$ $62 \%$<smiles>Cc1c(C(F)(F)F)c2ccccc2n1C</smiles><smiles>Cn1c(=O)c2c(nc(C(F)(F)F)n2C)n(C)c1=O</smiles>

图 9 光电催化 $\mathrm{C}\left(\mathrm{sp}^{2}\right)$ - $\mathrm{H}$ 三氟甲基化反应

Figure 9 Electrophotocatalyzed trifluoromethylations of $\mathrm{C}\left(\mathrm{sp}^{2}\right)-\mathrm{H}$ bond

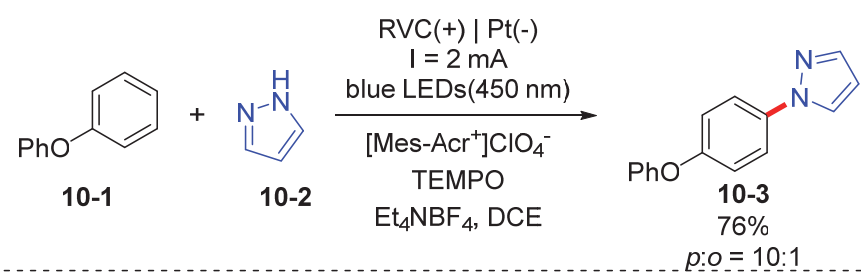

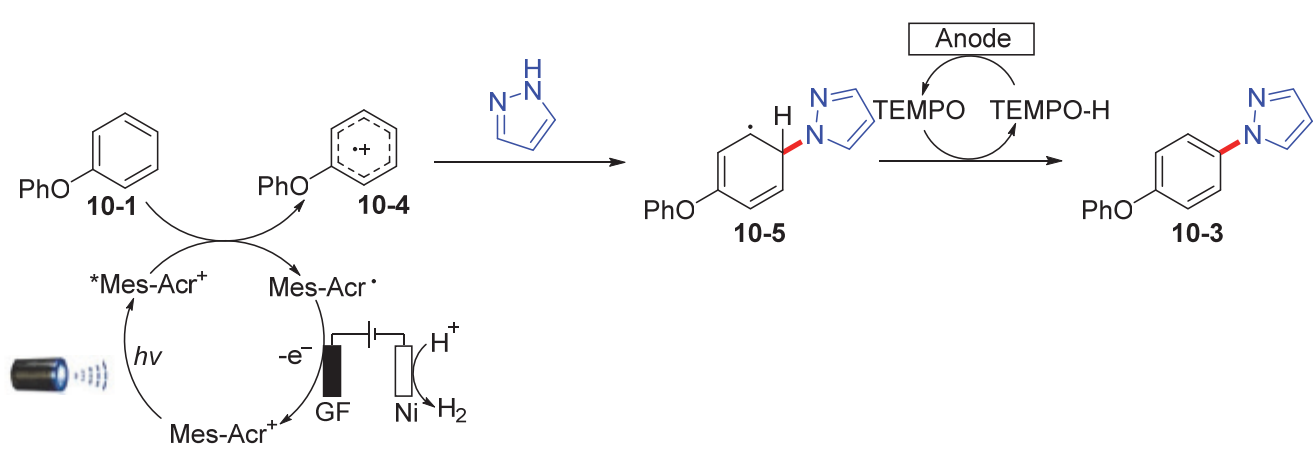<smiles>Cc1cc(C)c(-n2cccn2)c([N+](=O)[O-])c1</smiles><smiles></smiles><smiles>Cc1cc(C)c(-n2cc(Cl)cn2)c(C)c1</smiles><smiles>Cc1cc(C)c(-n2ccnc2)c([N+](=O)[O-])c1</smiles>

图 10 光电催化芳烃与唑类的交叉偶联反应

Figure 10 Electrophotocatalyzed cross-coupling of arenes and azoles

意的是, 在条件篮选中发现, 具有更强氧化性的 ${ }^{*} \mathrm{TAC}^{\cdot 2+}$ 应用于该反应时, 产率低于使用 DDQ, 由此可见, 催化 剂的氧化还原电势高低并不是 SET 反应能否发生的唯 一因素, 还需要和底物相匹配(图 11).
Lin 课题组 ${ }^{[38]}$ 等开发了一种核黄素四乙酸酯(RFT) 和硫嫝催化的醇氧化反应. 在催化循环中, RFT 催化剂 首先被光激发为 ${ }^{*} \mathrm{RFT}$, 并与硫嫝发生单电子转移(SET), 生成半醌形式的 $\left(\mathrm{RFT}^{*}\right)-\mathrm{H}$ 和自由基中间体 12-4. 然后 

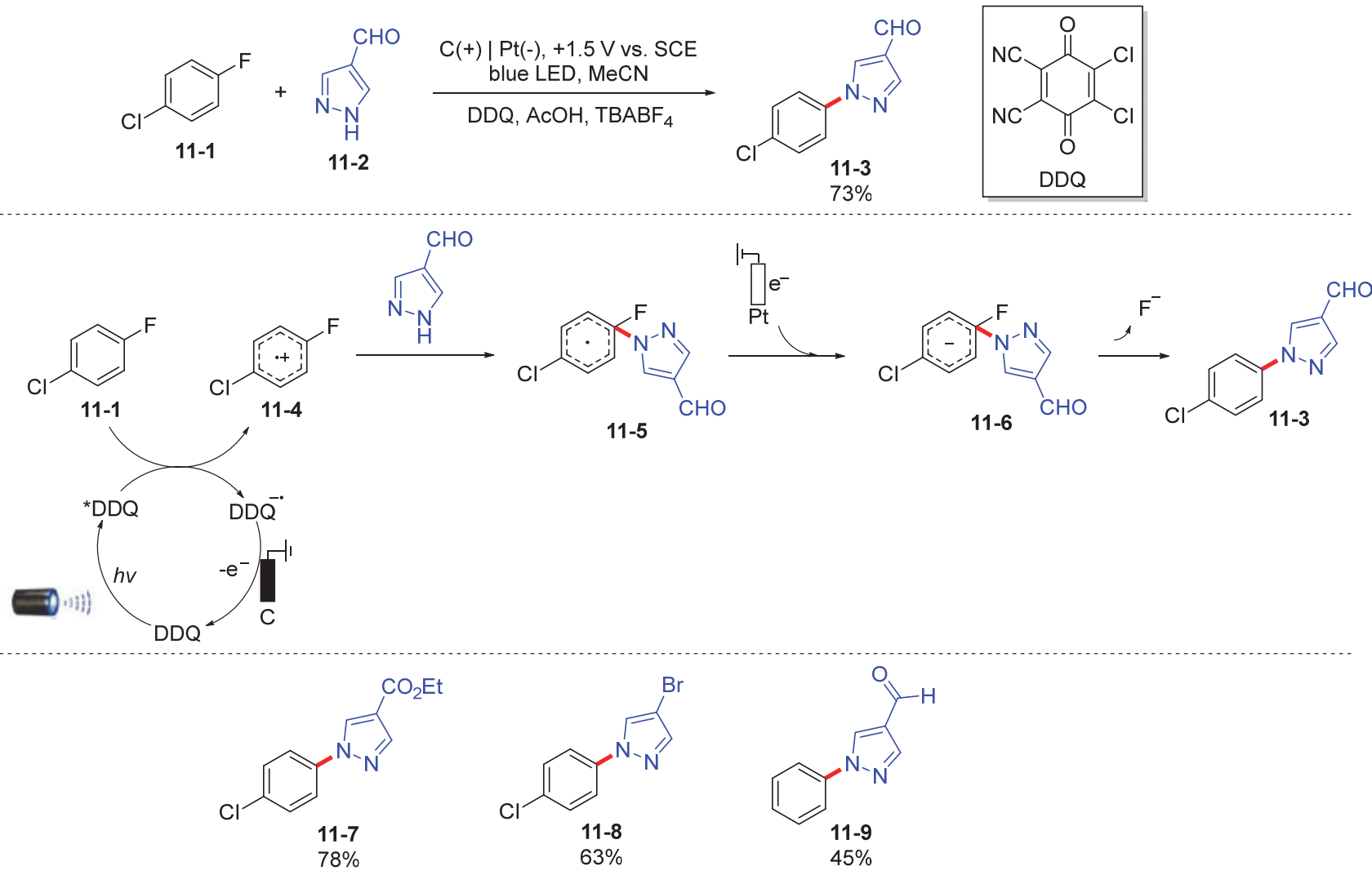

图 11 光电催化芳基氟化物的 $S_{N} A r$ 反应

Figure 11 Electrophotocatalyzed $\mathrm{S}_{\mathrm{N}} \mathrm{Ar}$ reactions of aryl fluorides

12-4 与醇底物进行氢原子转移(HAT)生成自由基中间体 12-5, 该中间体 12-5 可与 $\left(\mathrm{RFT}^{*}\right)-\mathrm{H}$ 再进行一次氢原子 转移(HAT)反应以生成羰基产物. 最后, (RFT) - $\mathrm{H}_{2}$ 在阳 极被氧化恢复为 RFT, 完成两个催化循环. 传统的核黄 素四乙酸酯/硫脲光催化氧化使用 $\mathrm{O}_{2}$ 作为末端氧化剂, 反应中会导致硫艮降解, 从而抑制反应产率, 且反应底 物范围仅限于茮醇. 该研究使用电化学氧化代替氧化剂 $\mathrm{O}_{2}$, 可以抑制催化剂的降解, 反应底物使用反应进一步 扩展到了未活化的脂肪族醇(图 12).

\section{3 解耦光电化学}

以上两类光电催化方法中, 光照和电流作用于同一 反应组分, 因此在一些综述中被统称为电化学介导光氧 化还原催化. 本节将要介绍一类光电反应, 在该类反应 中电化学和光化学独立地作用于不同物种, 被称为解耦 光电化学.

Stahl 课题组 ${ }^{[39]}$ 报道了在解耦光电化学条件下分子 内 $\mathrm{C}\left(\mathrm{sp}^{3}\right)-\mathrm{H}$ 键的 Hofmann-Löffler-Freytag (HLF)胺化反 应. 之前的电化学催化 HLF 反应需要较高的阳极电势, 因此官能团的耐受性受到了限制. 在光电催化的条件 下, 电化学生成的单质 $\mathrm{I}_{2}$ 在碱的存在下与甲苯磺胺底物 反应形成光化学不稳定的 N-I 中间体 13-3, 经过紫外 光照射, $\mathrm{N}$ - I 键发生均裂生成氮自由基中间体 13-4, 1,5-
氢迁移产生烷基自由基 13-5, 烷基自由基 13-5 被碘捕获 生成烷基碘中间体 13-6, 该中间体进一步通过氮亲核试 剂进行 Brønsted 碱促进的亲核取代完成 HLF 反应. 阳极 将碘化物氧化为分子碘. 该反应可以发生在活化以及非 活化的 $\mathrm{C}\left(\mathrm{sp}^{3}\right)-\mathrm{H}$ 键, 能耐受各种包括氧化不稳定的富 电子芳烃在内的带有杂环的底物. 与先前的电化学催化 HLF 反应相比, 此方法将碘化物氧化为分子碘所需的阳 极电位较低, 该电位比富电子芳烃和其他官能团的氧化 还原电位低, 因此在温和的条件下就可以实现氧化还原 反应, 在化学选择性方面显示出其关键优势. 同时 Stahl 还证明了先前报道的电子转移/质子转移/电子转移 (ET-PT-ET), 质子耦合电子转移(PCET)和溴化物介导的 电化学 HLF 反应均无法转化为产物, 而是生成了复杂 的混合物(图 13).

雷爱文课题组 ${ }^{[40]}$ 报道了锰催化的光电催化转化, 使用 $\mathrm{NaN}_{3}$ 作为亲核试剂, 对 $\mathrm{C}\left(\mathrm{sp}^{3}\right)-\mathrm{H}$ 键进行氧化叠氮 化的方法. 1,10-邻二氮杂菲 $(1,10-$-Phen $)$ 作为配体, DDQ 或者 9-芴酮(9-fluorenone)作为光敏剂. 机理研究表明, $\mathrm{NaN}_{3}$ 可以在阳极表面被直接氧化成叠氮自由基, $\mathrm{N}_{3}{ }^{-}$和 配体(1,10-Phen 或 $\mathrm{OAc}^{-}$等)与 $\mathrm{Mn}$ (II)配位并且发生阳极 氧化, 生成 $\mathrm{Mn}(\mathrm{III}) / \mathrm{L}-\mathrm{N}_{3}$ 中间体. 在光催化循环中, HAT 光催化剂被蓝色 LED 照射后激发引起了 $\mathrm{C}\left(\mathrm{sp}^{3}\right)-\mathrm{H}$ 

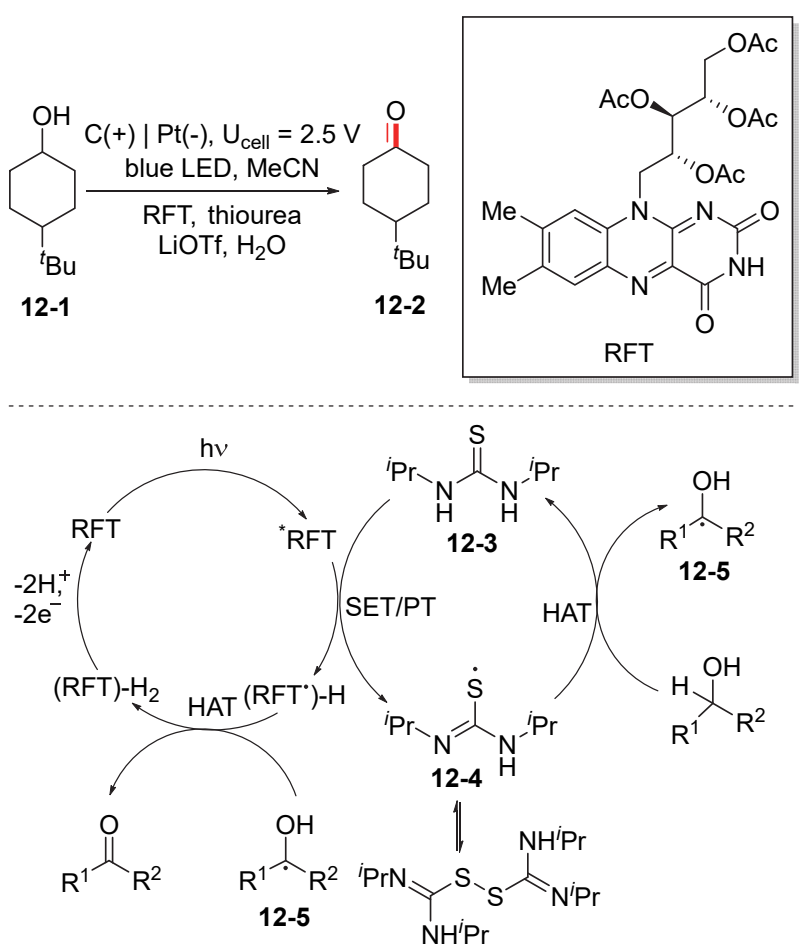

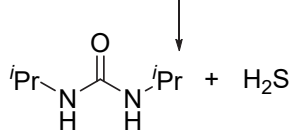

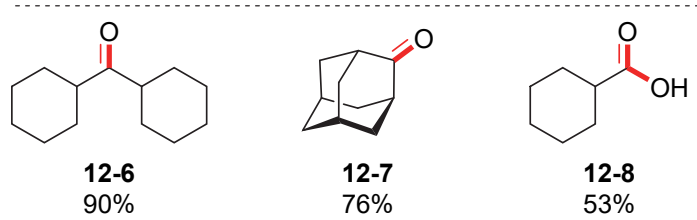

图 12 电化学调控的黄素催化光化学醇氧化

Figure 12 Electromediated flavin catalyzed photochemical oxidation of alcohols

键上的氢原子转移( $\mathrm{HAT}$ ), 从而生成 $\mathrm{C}\left(\mathrm{sp}^{3}\right)$ 自由基. 该光 催化剂的再生可以通过阳极氧化来完成. 同时, 叠氮化 物基团还可以㩲取 $\mathrm{C}\left(\mathrm{sp}^{3}\right)-\mathrm{H}$ 键的 $\mathrm{H}$ 原子以生成烷基自 由基. 最终, $\mathrm{Mn}(\mathrm{III}) / \mathrm{L}-\mathrm{N}_{3}$ 转移到烷基自由基形成烷基 叠氮化物，同时完成 $\mathrm{Mn}(\mathrm{II})$ 催化剂的循环(图 14).

徐海超课题组 ${ }^{[41]}$ 开发了杂芳烃与草酸烷基酯的无 氧化剂和无金属催化的光电催化 $\mathrm{C}-\mathrm{H}$ 烷基化反应. 该 光电化学反应采用 4CzIPN [2,4,5,6-四(9-咔唑基)-间苯 二腈]作为光催化剂, 无需牺牲化学氧化剂即可实现氧 化转化. 反应经历了如下过程: 首先草酸烷基酯 ( ${ }^{t} \mathrm{BuO}-$ $\mathrm{COCO}_{2} \mathrm{Cs}, E_{\mathrm{p}}^{\mathrm{ox}}=1.28 \mathrm{~V}$ vs. SCE) 与光激发的催化剂 ${ }^{*} 4 \mathrm{CzIPN}\left(E_{\mathrm{red}}=1.35 \mathrm{~V}\right.$ vs. SCE) 之间发生单电子转移 (SET)氧化, 产生持久的自由基阴离子 $4 \mathrm{CzIPN}^{-}$和双脱 羧后的烷基自由基 $\mathrm{R}^{*} . \mathrm{R}$ ·与质子化的杂环加成会生成自 由基阳离子 15-4, 该自由基阳离子会与 $4 \mathrm{CzIPN}^{-}$发生单 电子转移(SET)还原, 从而生成 1,2-二氢喹啉 15-5, 同时 伴随着基态的催化剂 $4 \mathrm{CzIPN}$ 的再生 $\left(E_{\mathrm{red}}=-1.21 \mathrm{~V}\right.$ vs.

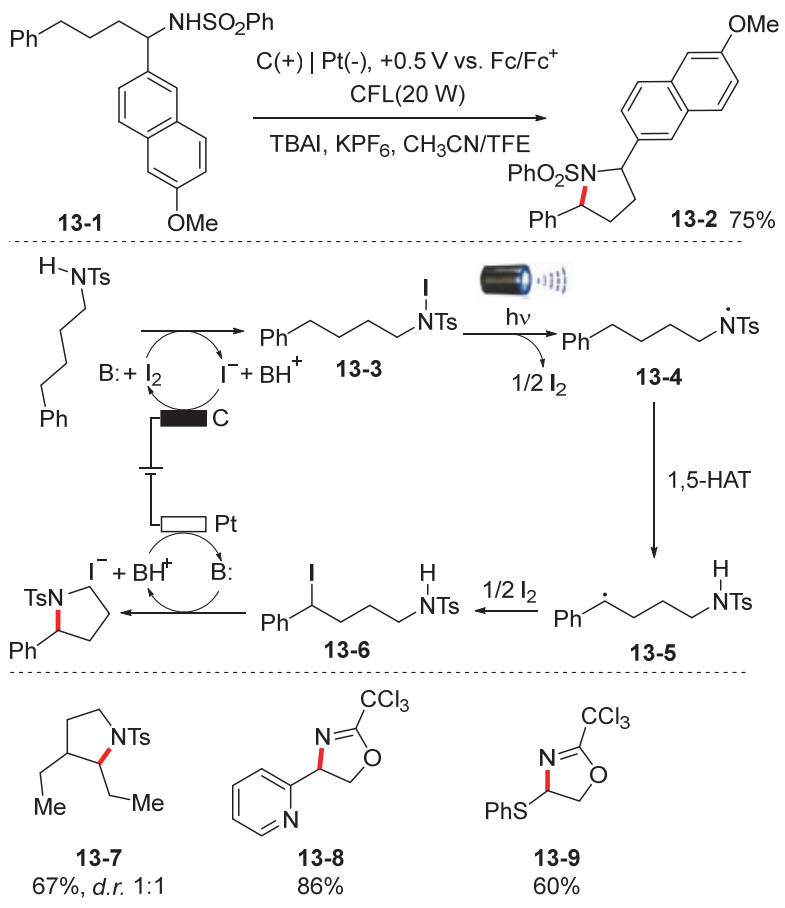

图 13 光电催化的分子内 $\mathrm{C}\left(\mathrm{sp}^{3}\right)-\mathrm{H}$ 键 $\mathrm{HLF}$ 反应

Figure 13 Electrophotocatalyzed intramolecular $\mathrm{C}\left(\mathrm{sp}^{3}\right)-\mathrm{H}$ HLF reaction

SCE). 15-5 阳极氧化生成最终的烷基化杂环 15-3. 质子 在阴极被还原产生 $\mathrm{H}_{2}$, 无需牺牲电子和质子受体(图 15).

\section{4 界面光电化学}

光电化学电池作为一种利用太阳能进行水分解以 生成氢气的装置近年来成为研究人员关注的焦点. 在光 电化学电池中, 光电极涂有光响应材料, 可以在光照下 产生强氧化或还原活性, 因此也适用于催化有机分子的 氧化还原反应. 这一类在光电电极表面上发生的有机反 应在一些综述中被称为界面光电化学.

Sammis 和 Berlinguette 课题组 ${ }^{[42]}$ 报道了一种界面光 电催化的四氢化萗的氧化反应, $\mathrm{BiVO}_{4}$ 被用作光电阳极 材料, 光源由模拟太阳光的装有滤光片的 $100 \mathrm{~W} \mathrm{Xe}$ 灯 提供. 反应中添加了 $N$-差基琥珀酰亚胺(NHS)作为一种 光电阳极和底物之间可溶且透明的空穴转移媒介. 对于 四氢化荎和环已烯的氧化, 必须使用 ${ }^{t} \mathrm{BuOOH}$ 作为外部 氧源. 当使用玻璃碳阳极/阴极的情况下，仅在电化学条 件 $(E=+1.8 \mathrm{~V}$ vs. $\mathrm{Ag} / \mathrm{AgCl})$ 下也可以实现相同的氧化反 应. 该界面光电催化方法可以在比电化学电池低 $1.0 \mathrm{~V}$ 的电压下工作, 相较于纯电化学预计可节能 $60 \%$. 尽管 产物的产量不高，但作者指出，由于有机产品的价值较 高, 以太阳能发电的效率 $(\eta=1.3 \%)$ 能够接近传统光电 化学水氧化法 $(\eta=1.7 \%)$ 的效率进行有机合成的能力十 分重要(图 16). 

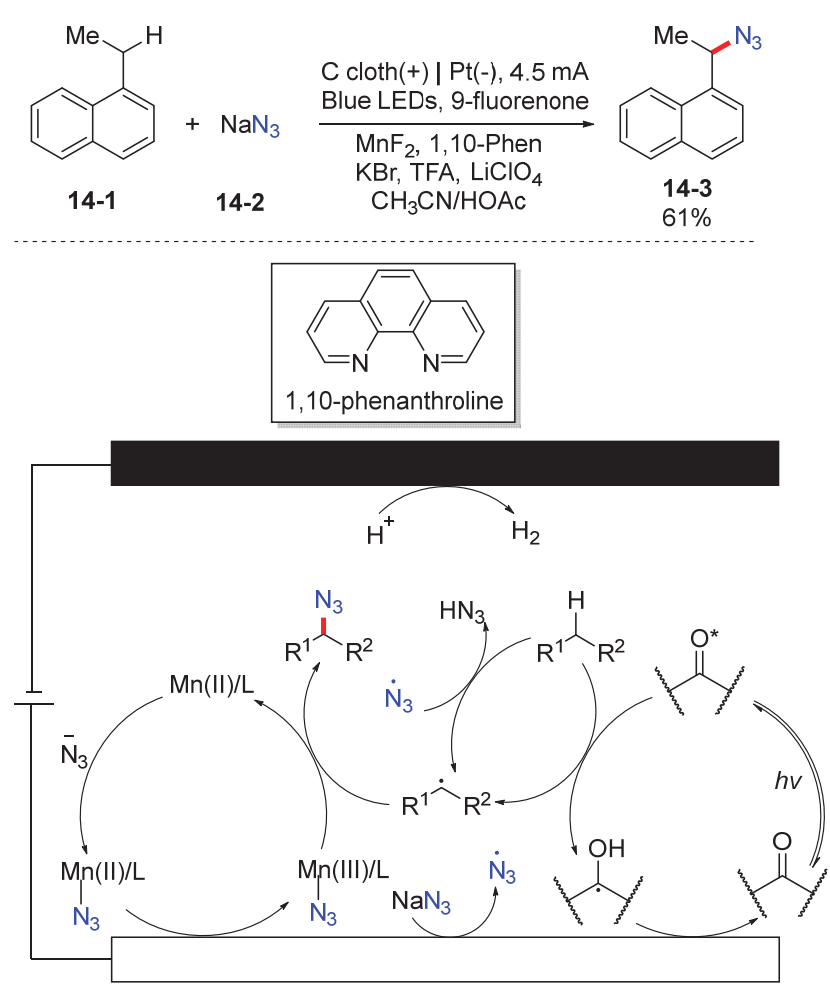

图 14 光电参与的锰催化 $\mathrm{C}\left(\mathrm{sp}^{3}\right)-\mathrm{H}$ 氧化叠氮化

Figure 14 Electrophoto- and Mn-catalyzed $\mathrm{C}\left(\mathrm{sp}^{3}\right)-\mathrm{H}$ oxidative azidation
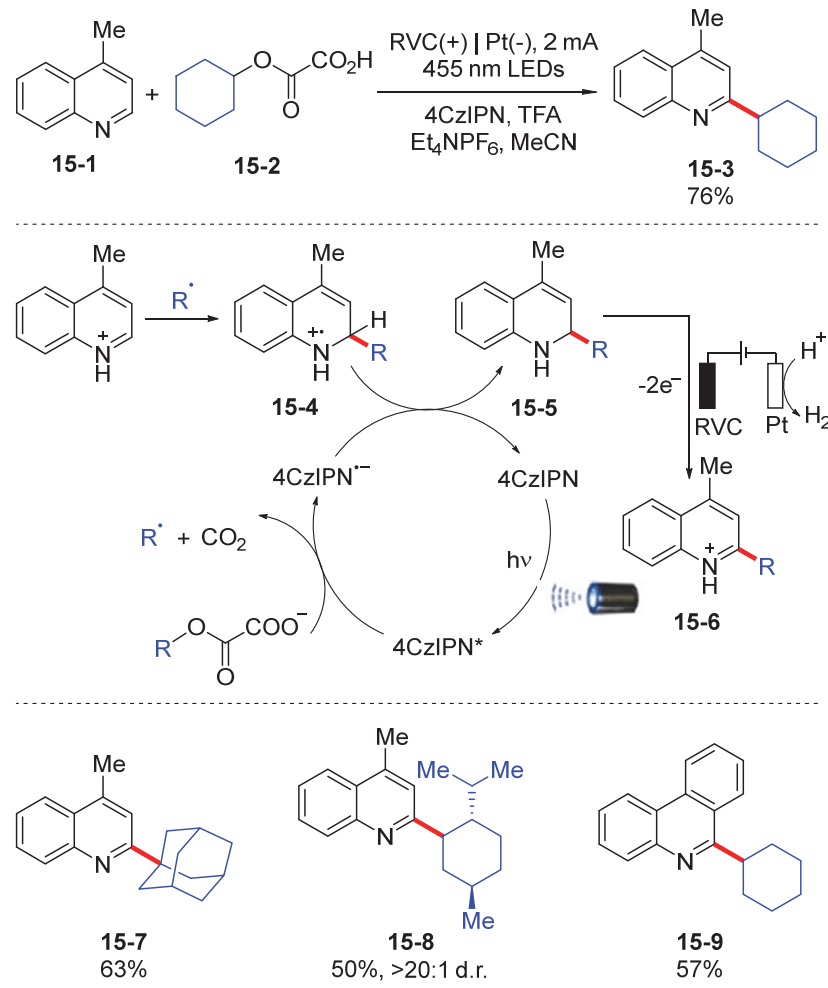

图 15 光电催化杂环芳烃与草酸烷基酯的 $\mathrm{C}-\mathrm{H}$ 烷基化反应 Figure 15 Electrophotocatalyzed $\mathrm{C}-\mathrm{H}$ alkylation of heteroarenes with alkyl oxalates

在与该研究类似的另一篇报道中, Sayama 课题

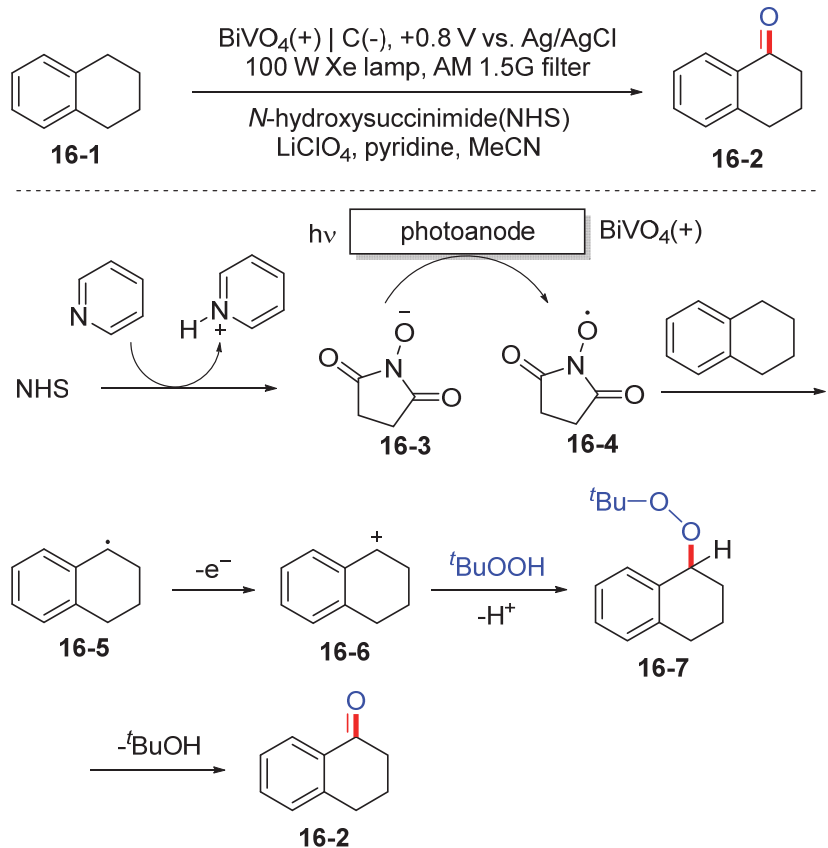

图 $16 \mathrm{BiVO}_{4}$ 光电阳极上的 $\mathrm{C}\left(\mathrm{sp}^{3}\right)-\mathrm{H}$ 键氧化反应

Figure 16 Oxidation of $\mathrm{C}\left(\mathrm{sp}^{3}\right)-\mathrm{H}$ bond on $\mathrm{BiVO}_{4}$ photoanode 组 ${ }^{[43]}$ 开发了一种 $\mathrm{WO}_{3} / \mathrm{BiVO}_{4}$ 光电阳极对呋喃的氧化方 法. 当光存在时, 两者氧化所施加的电势都会急剧下降. 在由溴离子介导的呋喃的光电氧化二甲氧基化中, 进一 步使用了 $\mathrm{WO}_{3} / \mathrm{BiVO}_{4}$ 光电阳极 ${ }^{[44]}$. 反应经过如下步骤: 首先通过光阳极氧化溴阴离子得到溴阳离子, 在传递了 $5 \mathrm{C}$ 的电荷并添加呋喃后, 以较高的收率获得了二甲氧 基化产物(图 17).

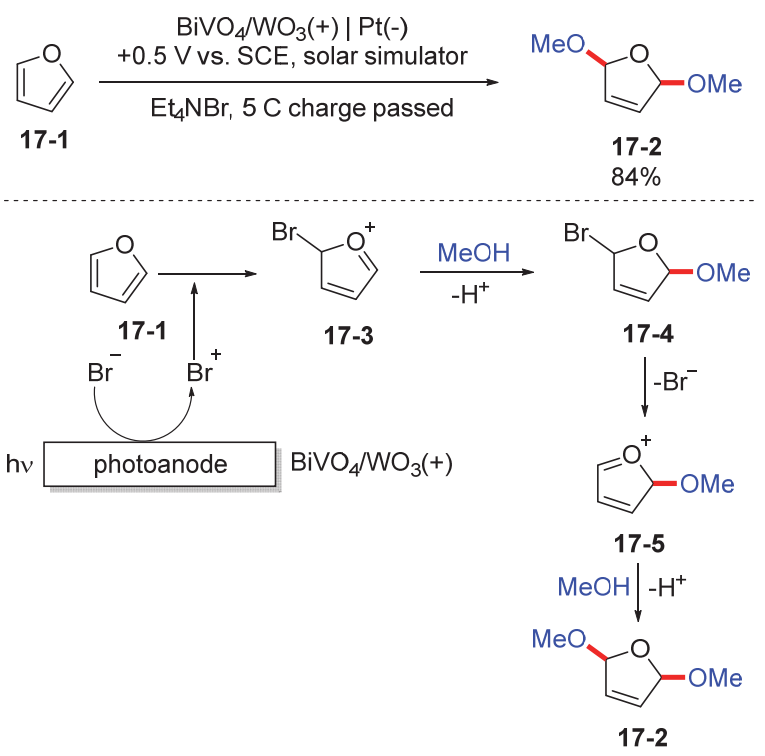

图 $17 \mathrm{WO}_{3} / \mathrm{BiVO}_{4}$ 光电阳极上的呋喃氧化二甲氧基化

Figure 17 Oxidative dimethoxylation of furans on $\mathrm{WO}_{3} / \mathrm{BiVO}_{4}$ photoanode

除了 $\mathrm{WO}_{3} / \mathrm{BiVO}_{4}$, 在地壳中含量丰富的赤铁矿 $\left(\alpha-\mathrm{Fe}_{2} \mathrm{O}_{3}\right)$ 也可用于发生界面光电催化反应. 2019 年, $\mathrm{Hu}$ 
课题 ${ }^{[45]}$ 报道了利用赤铁矿作为催化剂, 通过光电催化 的方法实现无导向基团参与芳烃 $\mathrm{C}-\mathrm{H}$ 键的胺化反应. 赤铁矿作为光电阳极, 在固定电势 $(+1.13 \mathrm{~V}$ vs. SCE $)$ 下 被蓝色 LED 的照射后在价带产生空穴, 被高度氧化(价 带 $=+2.30 \mathrm{~V}$ vs. SCE). 富电子的苯甲醚在阳极被氧化 成其自由基阳离子 18-4, 唑类化合物对其进行亲核进 攻, 生成中间体 18-5. 18-5 失去一个质子生成中间体 18-6, 18-6 被氧化后得到目标产物 18-3. 反应具有较好 的邻位选择性, 作者推测是由于苯甲醚、溶剂 HFIP 和 吡唑之间形成了氢键网络, 氢键的形成有助于在邻位发 生反应. 芳烃底物的范围仅限于富电子的芳烃. 在没有 光照的情况下, 需要较高的施加电势 $(+1.93 \mathrm{~V} \mathrm{vs.} \mathrm{SCE})$ 才能获得所需的化学物质, 但是产率下降. 当使用导电 玻璃碳电极 $(+1.73 \mathrm{~V}$ vs. SCE)直接电解会产生较差的 收率(图 18).
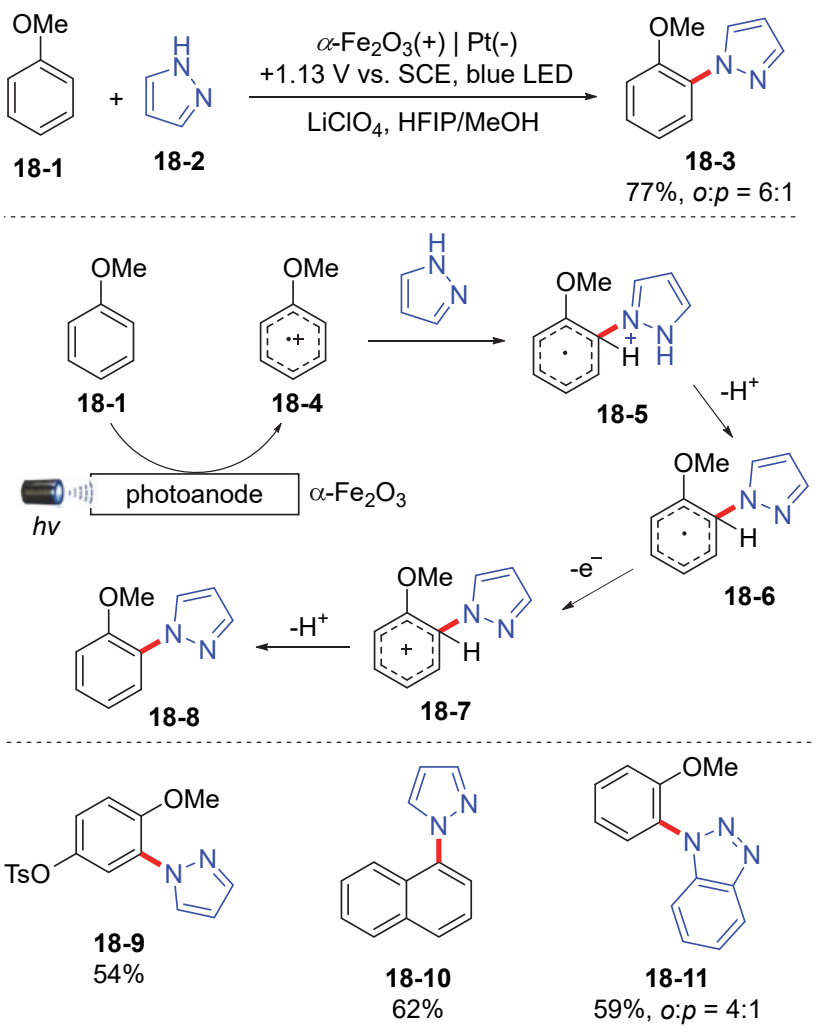

图 18 赤铁矿光电阳极上的芳烃 $\mathrm{C}-\mathrm{H}$ 键的胺化反应

Figure $18 \mathrm{C}-\mathrm{H}$ bond amination of arenes on hematite photoanode

由以上报道可以看出, 界面光电催化相较于传统电 化学的优势是可以降低施加的电势, 从而节约能源, 同 时较低的电势也避免了一些副反应的发生. 并且界面光 电催化不依赖于反应物的吸光性, 可以直接与不吸收可 见光的底物发生反应. 尽管到目前为止, 界面光电催化 只应用了少数简单的化学转化中, 但未来界面光电催化 在更复杂的底物的氧化还原转化中具有非常大的潜力.

\section{5 流动光电化学}

近几年, 流动化学作为一种新兴的技术在实验室和 工业界蓬勃发展, 目前已经有一些关于流动化学合成的

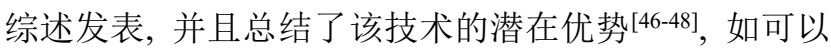
高度控制反应变量, 增加安全性, 可能实现反应自动化, 减少人工处理，重现性更高，灵活调控生产量，增加多 个反应相之间的接触面，在工厂中的占地面积更小等. 相较于传统在反应瓶进行的反应, 在工业应用中具有无 可比拟的优势.

光照和外加电流作为两种易于调控的反应条件, 非 常适合应用于流动化学 ${ }^{[49]}$. 最近已经有课题组验证了 光电催化在流动合成中的应用. Ackermann 课题组 ${ }^{[35]}$ 使 用 Mes-Acr ${ }^{+}$催化剂, 在碳毡阳极和镍阴极的模块化电 池和光照射的环形透明管搭建的流动化学体系中, 可以 高效合成目标产物(图 19).

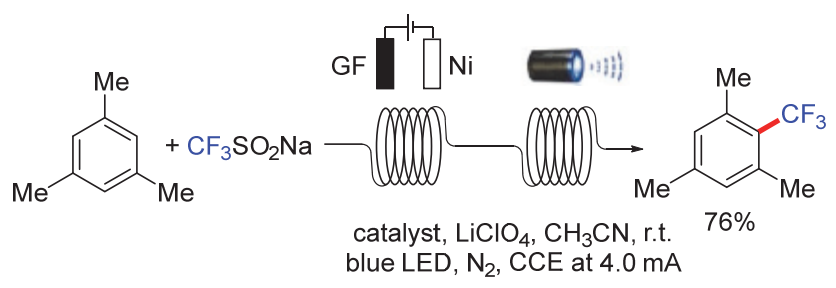

图 19 流动光电催化芳烃三氟甲基化反应

Figure 19 Continuous-flow electrophotochemical trifluoromethylations of arenes

徐海超课题组 ${ }^{[50-51]}$ 报道了一种两步连续流动系统, 通过对听啶核的芳基 $\mathrm{C}-\mathrm{H}$ 选择性官能团化来合成听啶 光催化剂. 通过连续洜使吅啶染料母核通过光反应器来 实现其与有机三氟硼酸盐的交叉偶联, 再通过电化学反 应器完成脱氢, 实现烷基化. 这种两步自动系统可以在 听啶染料的 3 位引入不同的烷基基团. 将 3-烷基化吟啶 盐再一次通过该烷基化的流动体系形成 3,6-二取代唤啶 (图 20).

\section{2 总结展望}

在过去的十年中, 随着光催化和有机电化学的复 兴, 光电化学也受到了科学家们的广泛关注并迅速兴 起. 这种协同催化方法的主要特点是在同一个反应中结 合电化学和光化学的优势, 广泛应用于不同反应类型. 最常见的反应过程是电氧化还原和光激发作用于同一 个反应物，生成所需的高反应性中间体. 另外一种常见 的反应过程是, 电氧化还原和光激发分别作用于两个离 散的反应组分, 继而完成反应. 光化学与电化学融合具 有以下优势: (1)扩大单电子转移化学的氧化还原窗口; (2)条件更温和, 相较传统的有机催化具有更好的官能 


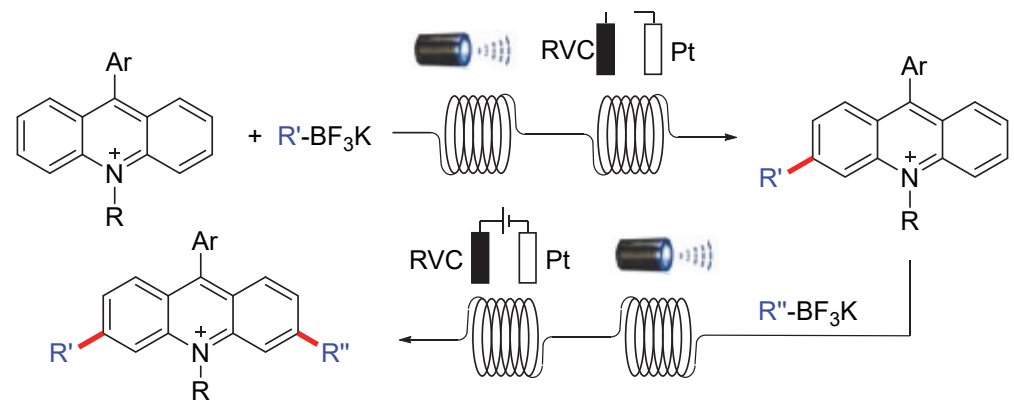

图 20 流动光电化学 $\mathrm{C}-\mathrm{H}$ 烷基化合成鲐啶光催化剂

Figure 20 Continuous-flow electrophotochemical C $-\mathrm{H}$ alkylation for the synthesis of acridinium photocatalysts

才耐受性和化学选择性; (3)原子经济性好; (4)流动光电 化学使工业化合成成为可能.

光电催化同时面临着一系列挑战，在未来的研究 中, 光电化学可以更加专注于传统化学难以实现或者无 法实现的化学转化; 设计合成新型光电催化剂以实现更 丰富的反应类型; 适用于界面光电催化的光电阳极材料 的开发也是未来研究重点之一; 设计搭建适用于光电联 用反应的反应器; 同时对流动光电化学体系进行优化, 通过与自动纯化和表征设备的联用, 积极开发人工智能 在光电催化反应中的应用, 实现光电催化反应的自动 化, 积极促进光电化学的工业化研究.

\section{References}

[1] Ciamician, G. Science 1912, 36, 385.

[2] Ischay, M. A.; Anzovino, M. E.; Du, J.; Yoon, T. P. J. Am. Chem. Soc. 2008, 130, 12886.

[3] Prier, C. K.; Rankic, D. A.; MacMillan, D. W. C. Chem. Rev. 2013, $113,5322$.

[4] Lu, Z.; Yoon, T. P. Angew. Chem. Int. Ed. 2012, 51, 10329.

[5] Yuan, Z. G.; Wang, Q.; Zheng, A.; Zhang, K.; Lu, L. Q.; Tang, Z.; Xiao, W. J. Chem. Commun. 2016, 52, 5128.

[6] Twilton, J.; Le, C.; Zhang, P.; Shaw, M. H.; Evans, R. W.; MacMillan, D. W. C. Nat. Rev. Chem. 2017, 1, 1.

[7] Xuan, J.; Xia, X. D.; Zeng, T. T.; Feng, Z. J.; Chen, J. R.; Lu, L. Q.; Xiao, W. J. Angew. Chem. Int. Ed. 2014, 53, 5653.

[8] Wu, H.; Mei, L.; Li, Y.; Chen, J. Chin. J. Org. Chem. 2019, 39, 3040 (in Chinese).

(陈锦杨, 李玉涵, 梅兰, 吴红谕, 有机化学, 2019, 39, 3040.)

[9] Chen, J. R.; Hu, X. Q.; Lu, L. Q.; Xiao, W. J. Acc. Chem. Res. 2016, 49, 1911 .

[10] Zhang, L.; Niu, C.; Yang, X.; Qin, H.; Yang, J.; Wen, J.; Wang, H. Chin. J. Org. Chem. 2020, 40, 1117(in Chinese).

(张龙菲, 牛联, 杨晓婷, 秦宏云, 杨建静, 文江伟, 王桦, 有机 化学, 2020, 40, 1117.)

[11] Weng, J.; Ye, F.; Xu, W.; Kong, Y. Chin. J. Org. Chem. 2019, 39, 3065 (in Chinese). (孔瑶蕾, 徐雯秀, 叶飞霞, 翁建全, 有机化学, 2019, 39, 3065.)

[12] Yan, M.; Kawamata, Y.; Baran, P. S. Chem. Rev. 2017, 117, 13230.

[13] Qiu, Y.; Zhu, C.; Stangier, M.; Struwe, J.; Ackermann, L. CCS Chem. 2021, 3, 1529.

[14] Wiebe, A.; Gieshoff, T.; Mohle, S.; Rodrigo, E.; Zirbes, M.; Waldvogel, S. R. Angew. Chem. Int. Ed. 2018, 57, 5594.

[15] Sauermann, N.; Meyer, T. H.; Qiu, Y.; Ackermann, L. ACS Catal.
2018, 8, 7086.

[16] Yuan, Y.; Lei, A. W. Acc. Chem. Res. 2019, 52, 3309.

[17] Qiu, Y.; Kong, W.-J.; Struwe, J.; Sauermann, N.; Rogge, T.; Scheremetjew, A.; Ackermann, L. Angew. Chem. Int. Ed. 2018, 57, 5828.

[18] Wang, X.; Xu, X.; Wang, Z.; Fang, P.; Mei, T. Chin. J. Org. Chem. 2020, 40, 3738 (in Chinese)

(王向阳, 徐学涛, 王振华, 方萍, 梅天胜, 有机化学, 2020, 40, 3738.)

[19] Qiu, Y.; Stangier, M.; Meyer, T. H.; Oliveira, J. C. A.; Ackermann, L. Angew. Chem. Int. Ed. 2018, 57, 14179.

[20] Feng, E.; Hou, Z.; Xu, H. Chin. J. Org. Chem. 2019, 39, 1424 (in Chinese).

(冯恩祺, 侯中伟, 徐海超, 有机化学, 2019, 39, 1424.)

[21] Qiu, Y.; Tian, C.; Massignan, L.; Rogge, T.; Ackermann, L. Angew. Chem. Int. Ed. 2018, 57, 5818.

[22] Qiu, Y.; Scheremetjew, A.; Ackermann, L. J. Am. Chem. Soc. 2019, $141,2731$.

[23] Moutet, J.-C.; Reverdy, G. Tetrahedron Lett. 1979, 20, 2389.

[24] Moutet, J.-C.; Reverdy, G. J. Chem. Soc., Chem. Commun. 1982, 0, 654.

[25] Scheffold, R.; Orlinski, R. J. Am. Chem. Soc. 1983, 105, 7200.

[26] Barham, J. P.; Konig, B. Angew. Chem. Int. Ed. 2020, 59, 11732.

[27] Liu, J. J.; Lu, L. X.; Wood, D.; Lin, S. ACS Cent. Sci. 2020, 6, 1317.

[28] Yu, Y.; Guo, P.; Zhong, J.-S.; Yuan, Y.; Ye, K.-Y. Org. Chem. Front. 2020, 7, 131 .

[29] Huang, H.; Strater, Z. M.; Rauch, M.; Shee, J.; Sisto, T. J.; Nuckolls, C.; Lambert, T. H. Angew. Chem. Int. Ed. 2019, 58, 13318.

[30] Huang, H.; Strater, Z. M.; Lambert, T. H. J. Am. Chem. Soc. 2020, 142,1698

[31] Shen, T.; Lambert, T. H. Science 2021, 371, 620.

[32] Kim, H.; Kim, H.; Lambert, T. H.; Lin, S. J. Am. Chem. Soc. 2020, 142, 2087

[33] Yan, H.; Hou, Z. W.; Xu, H. C. Angew. Chem. Int. Ed. 2019, 58, 4592.

[34] Xu, P.; Chen, P. Y.; Xu, H. C. Angew. Chem. Int. Ed. 2020, 59, 14275 .

[35] Qiu, Y. A.; Scheremetjew, A.; Finger, L. H.; Ackermann, L. Chem.Eur. J. 2020, 26, 3241.

[36] Hou, Z. W.; Xu, H. C. ChemElectroChem 2021, 8, 1571.

[37] Huang, H.; Lambert, T. H. Angew. Chem. Int. Ed. 2020, 59, 658

[38] Zhang, W.; Carpenter, K. L.; Lin, S. Angew. Chem. Int. Ed. 2020, $59,409$.

[39] Wang, F.; Stahl, S. S. Angew. Chem. Int. Ed. 2019, 58, 6385.

[40] Niu, L.; Jiang, C.; Liang, Y.; Liu, D.; Bu, F.; Shi, R.; Chen, H.; Chowdhury, A. D.; Lei, A. J. Am. Chem. Soc. 2020, 142, 17693.

[41] Xu, H.-C.; Xu, F.; Lai, X.-L. Synlett 2020, 32, 369.

[42] Li, T. F.; Kasahara, T.; He, J. F.; Dettelbach, K. E.; Sammis, G. M.; 
Berlinguette, C. P. Nat. Commun. 2017, 8, 1.

[43] Tateno, H.; Miseki, Y.; Sayama, K. ChemElectroChem 2017, 4, 3283.

[44] Tateno, H.; Miseki, Y.; Sayama, K. Chem. Commun. 2017, 53, 4378.

[45] Zhang, L.; Liardet, L.; Luo, J.; Ren, D.; Grätzel, M.; Hu, X. Nat. Catal. 2019, 2, 366.

[46] Malet-Sanz, L.; Susanne, F. J. Med. Chem. 2012, 55, 4062.
[47] Britton, J.; Raston, C. L. Chem. Soc. Rev. 2017, 46, 1250.

[48] Cambie, D.; Bottecchia, C.; Straathof, N. J. W.; Hessel, V.; Noel, T. Chem. Rev. 2016, 116, 10276.

[49] Elsherbini, M.; Wirth, T. Acc. Chem. Res. 2019, 52, 3287.

[50] Yan, H.; Zhu, S.; Xu, H.-C. Org. Process Res. Dev. 2021. doi. org/10.1021/acs.oprd.1c00038.

[51] Yan, H.; Song, J.; Zhu, S.; Xu, H.-C. CCS Chem. 2021, 3, 317.

(Fan, Y.) 